BULLETIN OF THE

AMERICAN MATHEMATICAL SOCIETY

Volume 80, Number 1, January 1974

\title{
MOTIONS OF LINKS IN THE 3-SPHERE
}

\author{
BY DEBORAH LOUISE GOLDSMITH
}

Communicated by William Browder, May 24, 1973

1. Introduction. A motion in $M$ of a subspace $N$ consists of an (ambient) isotopy of $N$ through $M$ which ultimately returns $N$ to itself. Here we study the problem of determining all essentially different motions and the natural group structure on this set which is induced when two motions are multiplied by performing them on $N$ in succession.

The aim of this paper is to calculate the group of motions of links in the 3 -sphere and in 3-space. In $\$ 3$ this is reduced, for "links with generalized axis", to a calculation of isotopy classes of homeomorphisms of a surface punctured in a finite number of points. In $\S 4$, generators are given for the motion group of a torus link in $S^{3}$, and generators and relations are given for the motion group of a torus knot in $\boldsymbol{R}^{3}$ (in fact I can give generators and relations for both groups).

We begin with a definition of motion groups based on Dahm's original definition:

2. Motion groups. Let $M$ be a manifold, $N$ a subspace contained in the interior of $M$. Denote by $H(M)$ the group of autohomeomorphisms of $M$ with the compact open topology, where if $M$ has boundary $\partial M$, all homeomorphisms are required to fix $\partial M$ pointwise; and let $H(M ; N)$ be the subgroup of maps restricting to an autohomeomorphism of $N$, with the subspace topology. The notation $\mathscr{H}(M)$ and $\mathscr{H}(M ; N)$ is used for the group of path components of $H(M)$ and $H(M ; N)$, respectively. Denote the identity map of $M$ by $1_{M}: M \rightarrow M$.

A motion of $N$ in $M$ is a path $f_{t}$ in $H(M)$ beginning at $f_{0}=1_{M}$ and ending at $f_{1}$ where $f_{1}(N)=N$. The motion $f$ is said to be a stationary motion of $N$ in $M$ if $\forall t, f_{t}(N)=N$. To compose two motions, translate the second by multiplication in the group $H(M)$ so that its initial endpoint coincides with the terminal endpoint of the first, and multiply as in the groupoid of paths. Define the inverse $f^{-1}$ of a motion $f$ to be the inverse path of the path $f$ in $H(M)$, translated so that its initial endpoint is $1_{M}$. Finally, we say that the motions $f$ and $g$ are equivalent if the path $g^{-1} \circ f$ is homotopic modulo its endpoints to a stationary motion. Thus stationary motions of $N$ in $M$ are to be considered trivial; i.e., they are equivalent to the trivial path mapping to the point $1_{M}$. The group of motions of $N$ in $M$, denoted

AMS (MOS) subject classifications (1970). Primary 55A25. 
$\mathscr{M}(M ; N)$, is the set of equivalence classes of motions of $N$ in $M$ with multiplication induced by composition of motions.

To illustrate, we remark that the group of motions of an interior point in a manifold $M$ is the group $\pi_{1}(M)$, and that the group of motions of $n$ distinct points in the interior of a 2-disk is the $n$th braid group $B_{n}$.

More generally, let $\mathscr{H}\left(M ; N_{1}, \cdots, N_{n}\right)$ denote the group of path components of the space $H\left(M ; N_{1}\right) \cap \cdots \cap H\left(M ; N_{n}\right)$ of autohomeomorphisms of $M$ which restrict to autohomeomorphisms of $N_{i}$ for all $i$, and analogously define the group $\mathscr{M}\left(M ; N_{1}, \cdots, N_{n}\right)$ of motions of the $n$-tuple of subspaces $\left(N_{1}, \cdots, N_{n}\right)$ in $M$, where each $N_{i}$ is returned to itself by these motions.

3. Motions of links in $S^{3}$. The most obvious motion of a link $L$ in $R^{3}$ or $S^{3}$ is the $2 \pi$ rotation, written $2 \pi$. This consists of rotating a 3-ball containing the link and centered at the origin by $2 \pi$ radians about the $z$-axis. The class $\{2 \pi\}$ either is trivial or has order two in the motion group $\mathscr{M}\left(S^{3} ; L\right)$, and it always has order two in the motion group $\mathscr{M}\left(\boldsymbol{R}^{3} ; L\right)$ provided the link $L$ is nontrivial.

The next most obvious collection of motions arises from moving a link in the complement of one of its axes. Since an axis is an unknotted simple closed curve about which the link $L$ winds, the intersection of $L$ with a suitable disk which the axis bounds is a finite set of points; it is natural to ask when a motion of these points in the disk will extend to a motion of $L$ in the complement of its axis, whether nontrivial motions of the points can extend to trivial motions of $L$, and which motions of $L$ can be performed in the complement of an axis. These questions motivate the following definition and theorems.

Definition. The simple closed curve $A$ in $S^{3}-L$ is said to be a generalized axis for the link $L$ if

(1) $S^{3}-A$ fibers over the circle with fiber homeomorphic to the interior of a surface $S$ in $S^{3}$ with boundary $A$;

(2) The surface $S$ may be chosen so that the intersection $S \cap L$ of the link with $S$ is a finite set of points $P$;

(3) the fibration is given by the map $f:[0,1] \times S \rightarrow S^{3}$ satisfying

(i) $f_{t}(A)=A$ and $f_{t}: A \rightarrow A$ is a homeomorphism for all $t \in[0,1]$,

(ii) $f_{0}: S \rightarrow S$ is the identity and $f_{1}: S \rightarrow S$ is a homeomorphism,

(iii) $f \mid(0,1) \times(S-A)$ is a homeomorphism onto $S^{3}-S$,

(iv) $f([0,1] \times P)=L$.

An axis for $L$ is simply a generalized axis which is unknotted.

We may assume without loss of generality that the map $f_{1}:(S, P) \rightarrow$ $(S, P)$ is the identity on the boundary $A$ of $S$. Denote the map $f_{1}$ in $H(S ; P)$ by $\Sigma$, and let $\mathscr{H}_{\Sigma}(S ; P)$ be the centralizer of $\{\Sigma\}$ in $\mathscr{H}(S ; P)$. 
Define the subgroup $\mathscr{M}_{\Sigma}(S ; P)$ of motions of $P$ in $S$ to consist of classes of those motions $f$ such that $\Sigma^{-1} \circ f_{t} \circ \Sigma$ defines a motion equivalent to $f$. If $S$ is a disk, then there is a unique motion class $\sigma$ whose terminal endpoint is a representative of the class $\{\Sigma\}$. We now have:

THEOREM 1a. The sequence

$$
1 \rightarrow \mathscr{M}_{\Sigma}(S ; P) \rightarrow \mathscr{M}\left(S^{3} ; L, A\right) \rightarrow \mathscr{M}\left(S^{3} ; A\right)
$$

is exact if $S$ is not a disk.

THEOREM 1b. If $S$ is a disk (and hence $A$ is an axis of $L$ ), the sequence

$$
\mathscr{M}_{\Sigma}(S ; P) \rightarrow \mathscr{M}\left(S^{3} ; L, A\right) \rightarrow \mathscr{M}\left(S^{3} ; A\right)=Z_{2}
$$

is exact, and the kernel of the map $\mathscr{M}_{\Sigma}(S ; P) \rightarrow \mathscr{M}\left(S^{3} ; L, A\right)$ is contained in the subgroup of $\mathscr{M}_{\Sigma}(S ; P)$ generated by $\sigma$ and $c$, the generator of the center of the braid group $\mathscr{M}(S ; P)$, where $\sigma, c \rightarrow\{2 \pi\}$.

THEOREM 2. The sequence

$$
\mathscr{H}_{\Sigma}(S ; P) \rightarrow \mathscr{M}\left(S^{3} ; L, A\right) \rightarrow Z_{2}
$$

is exact, and the kernel of the map $\mathscr{H}_{\Sigma}(S ; P) \rightarrow \mathscr{M}\left(S^{3} ; L, A\right)$ is contained in the subgroup generated by $\{\Sigma\}$ and $c$, the generator of the center of the braid group $\mathscr{H}(S ; P)$, where $c \rightarrow\{2 \pi\}$ and $\{\Sigma\} \rightarrow\{2 \pi\}$.

Discussion of Theorems 1 AND 2 . The exact sequences $(*)$ and (**) assert that the classes of motions of $P$ in $S$ which extend to motions of $L$ in the complement of the generalized axis $A$ comprise the subgroup $\mathscr{M}_{\Sigma}(S ; P)$ of $\mathscr{M}(S ; P)$, where the homomorphism induced from motions of $P$ in $S$ to motions of $(L, A)$ in $S^{3}$ is injective if $S$ is not a disk, and otherwise has image equal to all of $\mathscr{M}\left(S^{3} ; L, A\right)$ or a subgroup of $\mathscr{M}\left(S^{3} ; L, A\right)$ of index 2 . Theorems 1 and 2 are most useful when $L$ is the union of a sublink $L^{\prime}$ and a generalized axis $A$ of $L^{\prime}$. For these links with generalized axis it is often true that the motion groups $\mathscr{M}\left(S^{3} ; L\right)$ and $\mathscr{M}\left(S^{3} ; L^{\prime}, A\right)$ are the same; for example if $A$ has a different knot type from the components of $L$. In this case, the exact sequence $(* * *)$ calculates $\mathscr{M}\left(S^{3}, L\right)$ to be a specified quotient of the group $\mathscr{H}_{\Sigma}(S ; P)$ of homeomorphisms of a punctured surface, or a $Z_{2}$-extension thereof.

4. The group of motions of a torus link in $S^{3}$. A torus link is a link which may be embedded without self-intersections on the surface of a torus canonically embedded in $S^{3}$. A torus knot is a torus link having one component. Each such knot is of type $(p, q)$ for some relatively prime positive integers $p$ and $q$, where a $(p, q)$ torus knot wraps $p$ times in the direction of the longitude and $q$ times in the direction of the meridian. A type $(2,3)$ torus knot is illustrated in Figure 1. 


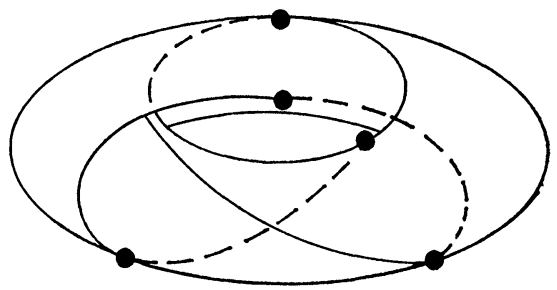

A $(2,3)$ torus knot

FIGURE 1

Every torus link is equivalent, by a deformation of $S^{3}$, to $k$ torus knots of the same type inscribed, respectively, on concentric tori of increasing radius canonically embedded in $S^{3}$. Numbering the tori in order of increasing radius, we say that the $i$ th torus knot lies on the $i$ th torus. If the type of each knot is $(p, q)$, the link is said to be a $(p, q, k)$ torus link.

The $i$ th torus associated with a $(p, q, k)$ torus link $L$ separates $S^{3}$ into two solid tori, whose core circles are called the canonical axes of $L$; the $p$-axis is that canonical axis linking the $i$ th torus knot $p$ times, the $q$-axis, the one linking the $i$ th knot $q$ times. These axes are independent of choice of $i$.

We will now begin to calculate the group of motions of a torus link in $S^{3}$. Let $L$ denote a $(p, q, k)$ torus link consisting of the $(p, q)$ torus knots $L_{1}, \cdots, L_{k}$; let $A_{p}$ denote the $p$-axis, $A_{q}$ the $q$-axis of $L$ :

THEOREM 3. The natural homomorphisms $\mathscr{M}\left(S^{3} ; L, A_{p}\right) \rightarrow \mathscr{M}\left(S^{3} ; L\right)$ and $\mathscr{M}\left(S^{3} ; L, A_{q}\right) \rightarrow \mathscr{M}\left(S^{3} ; L\right)$ induced by restricting motions of $L$ together with a canonical axis in $S^{3}$ to motions of $L$ in $S^{3}$, are surjective.

THEOREM 4. If $p \neq 1$, then the natural homomorphism $\mathscr{M}\left(S^{3} ; L, A_{q}\right) \rightarrow$ $\mathscr{M}\left(S^{3} ; L\right)$ induced by restricting motions of $\left(L, A_{q}\right)$ in $S^{3}$ to motions of $L$ in $S^{3}$ is an isomorphism. Similarly, $\mathscr{M}\left(S^{3} ; L, A_{p}\right) \rightarrow \mathscr{M}\left(S^{3} ; L\right)$ is an isomorphism if $q \neq 1$.

Proof SKetch of Theorems 3 AND 4. In preparation for the use of Theorem 2, let $L^{\prime}=L_{2} \cup \cdots \cup L_{k}$ and note that the torus knot $L_{1}$ is a generalized axis for both $L^{\prime}$ and $L^{\prime} \cup A_{p} \cup A_{q}$. Now if $S$ is a spanning surface for $L_{1}$ as in the definition of generalized axis, and $P_{q}=A_{q} \cap S$, $P_{p}=A_{p} \cap S$, the autohomeomorphism $\Sigma$ of $S$ is a periodic map of period $p q$ with singular orbits $P_{q}$ (on which $\Sigma$ has period $q$ ) and $P_{p}$ (on which $\Sigma$ has period $p$ ). Call a homeomorphism $f \in H(S)$ symmetric if $f$ commutes with $\Sigma$. Then the group $\mathscr{H}_{\Sigma}(S ; P)$ is naturally isomorphic to the group of isotopy classes (through symmetric maps) of symmetric maps. Hence the homomorphism $\mathscr{H}_{\Sigma}\left(S ; P, P_{q}\right) \rightarrow \mathscr{H}_{\Sigma}(S ; P)$ induced by inclusion is surjective, and in case $p \neq 1$, is an isomorphism. (This result is symmetric 
with respect to $p$ and $q$.) The above in conjunction with Theorem 2 provides most of the proof.

COROLlary 5. The motion group of a type $(p, q, k)$ torus link in $S^{3}$ is generated by the following motions:

(1) i-rotation: The ith concentric torus is rotated by $2 \pi / q$ radians on itself about its p-axis.

(2) ij-interchange: The ith torus knot is moved onto the jth concentric torus and the jth torus knot onto the ith concentric torus, so that the ith and jth torus knots are interchanged. A cross sectional view of this motion is the motion of the points of intersection of $L$ with a disk spanning the p-axis, shown in Figure 2.

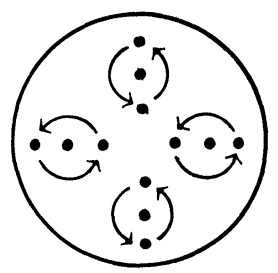

FIGURE 2

(3) flip: The p-axis of L is flipped, carrying each torus knot to itself with the reversed orientation. We use the invertibility of torus knots and links in the construction of this motion.

Corollary 6. Let $K$ be $a(p, q)$ torus knot in $\boldsymbol{R}^{3}$. The group $\mathscr{M}\left(\boldsymbol{R}^{3} ; K\right)$ of motions of $K$ in $\boldsymbol{R}^{3}$ is

$$
\begin{aligned}
&\left\{a, b, f_{1}\{a \pi\}: a^{p}=b^{a}=f^{2}=\{2 \pi\},\{2 \pi\}^{i}=1,\right. \\
&\left.f\{2 \pi\} f^{-1}=\{2 \pi\}, f a f^{-1}=a^{-1}, f b f^{-1}=b^{-1}\right\}
\end{aligned}
$$

where $i=1$ if either $p$ or $q$ is even, and $i=2$ if both $p$ and $q$ are odd; $f$ is the flip, the motion $a$ is the 1-rotation of Corollary 5, and $b$ is a composite $m^{-1} \circ r \circ m$ of a motion $m$ taking the $(p, q)$ torus knot to the $(q, p)$ torus $k n o t$, with the 1-rotation $r$ of the $(q, p)$ torus knot.

\section{REFERENCES}

1. D. Dahm, A generalization of braid theory, Ph.D. Thesis, Princeton University, Princeton, N.J., 1962.

2. F. Waldhausen, On irreducible 3-manifolds which are sufficiently large, Ann. of Math. (2) 87 (1968), 56-88. MR 36 \#7146.

Department of Mathematics, University of Chicago, Chicago, Illinois 60637 